\title{
Transbronchial Lung Cryobiopsy in Interstitial Lung Diseases: Best Practice
}

\author{
Sara Colella ${ }^{a}$ Maik Haentschel $^{b}$ Pallav Shah ${ }^{c-e}$ Venerino Polettif, $g$ \\ Jürgen Hetzel ${ }^{\mathrm{b}}$ \\ a Pulmonary Unit, Ospedale "C. e G. Mazzoni", Ascoli Piceno, Italy; ${ }^{b}$ Department of Hematology, Oncology, \\ Rheumatology, Immunology and Pulmonology, University of Tübingen, Tübingen, Germany; ${ }^{`}$ Royal Brompton \& \\ Harefield NHS Foundation Trust, London, UK; ${ }^{d}$ Chelsea and Westminster Hospital NHS Foundation Trust, London, \\ UK; ${ }^{e}$ National Heart \& Lung Institute, Imperial College, London, UK; ' Department of Diseases of the Thorax, \\ Ospedale GB Morgagni, Forlì, Italy; ${ }^{9}$ Department of Respiratory Diseases and Allergy, Aarhus University Hospital, \\ Aarhus, Denmark
}

\section{Keywords}

Transbronchial lung cryobiopsy · Interstitial lung disease · Bronchoscopy · Diffuse lung disease $\cdot$ Idiopathic pulmonary fibrosis

\begin{abstract}
The lung biopsy in interstitial lung disease (ILD) represents an important diagnostic step when the clinical and radiological data are insufficient for a firm diagnosis. A growing body of evidence suggests the utility of transbronchial lung cryobiopsy (TBLC) in the diagnostic algorithm of ILD as it allows, compared to transbronchial lung biopsy with conventional forceps, a better identification of complex histological patterns - such as usual interstitial pneumonia - and can provide information which has a clinical impact on the multidisciplinary discussion similar to that provided by surgical lung biopsy. Performed correctly, it appears to have a better safety profile than surgery. The decision to perform a lung biopsy should be a multidisciplinary decision process where it is felt that there is sufficient diagnostic doubt after a careful clinical evaluation including review of the computed tomograms of the thorax. The presence of severe pulmonary hypertension (>50 $\mathrm{mm} \mathrm{Hg}$ ), poor lung function (FVC <50\%), or dismissed gas transfer (DLCO of $<35 \%$ ) are considered rel-
\end{abstract}

ative contraindications for TBLC. Anticoagulants and antiplatelet drugs should be discontinued for the minimum period required for the specific drugs. The greatest consideration should be given to ensure the biopsy is performed safely and we recommend the use of either an endotracheal tube or rigid bronchoscopy. Deep sedation or general anesthesia allow better control of the procedure and a better patient experience. Prophylactic balloon blockers should be used to tamponade any bleeding and also to prevent overspill of blood from the segment that is being sampled. The procedure should be performed under fluoroscopy to ensure that samples are ideally obtained about $10 \mathrm{~mm}$ from the pleural edge. The cryoprobe is activated for about $5 \mathrm{~s}$ for the first biopsy and then adjusted according to the sample size obtained. With a careful standardized approach it is possible to obtain good-quality lung specimens for diagnosis in a safe manner.

(c) 2018 S. Karger AG, Basel

\section{S. Colella and M. Haentschel contributed equally.}

Previous articles in this series: 1. Shah PL, Herth FJF: Progress in Interventional Pulmonology. Respiration 2018;95:287-288. 2. Bonta PI, Chanez P, Annema JT, Shah PL, Niven R: Bronchial Thermoplasty in Severe Asthma: Best Practice Recommendations from an Expert Panel. Respiration 2018;95:289-300.

\section{KARGER}

(C) 2018 S. Karger AG, Basel

E-Mail karger@karger.com

www.karger.com/res 


\section{Introduction}

\section{Lung Biopsy in Interstitial Lung Disease:}

The Rationale

The diagnosis of interstitial lung diseases (ILDs) requires the integration of clinical and radiological data, and when there is not enough information to draw a diagnostic conclusion, supplementary morphological information from a lung biopsy is necessary.

The lung specimens may contain very specific and informative lesions (also using immunohistochemical staining) that could be diagnostic by themselves (e.g., carcinomatous lymphangitis, other neoplasms, lymphangioleiomyomatosis, or Langerhans cell histiocytosis). Alternatively, the pathology may not be diagnostic on its own but has specific informative patterns and therefore the integration with clinical and radiological data is necessary (e.g., organizing pneumonia, granulomas, hypersensitivity pneumonitis pattern). However, the lung biopsy may not be informative when the morphological findings are completely incongruous with the clinicalradiological context [1-4]. Several techniques for lung biopsy have been proposed in an attempt to get the greatest amount of information with the least invasive procedure. Other minimally invasive diagnostic techniques such as endobronchial fine needle aspiration (EBUSFNA) and endoesophageal ultrasound fine needle aspiration (EUS-FNA) have been proposed in those cases where an involvement of mediastinal lymph nodes is also present.

\section{Techniques for Lung Biopsy}

Invasive or semi-invasive procedures may increase the diagnostic accuracy in patients with suspected ILDs in which high-resolution computed tomography (HRCT) features are not characteristic, but this needs to be balanced with the risks the patients are dealing with when these procedures are carried out.

Bronchoalveolar lavage (BAL) has the least side effects. It may increase the diagnostic confidence when idiopathic pulmonary fibrosis (IPF) is the probable diagnostic hypothesis documenting a slight increase of neutrophils and eosinophils without a concomitant lymphocytosis. In some cases, the likelihood of alternative diagnoses is increased, e.g., in the case of chronic hypersensitivity pneumonitis when a lymphocytosis is documented. More rarely, an unexpected diagnosis may be noteworthy for the presence of characteristic features in BAL fluids, e.g., infectious diseases, alveolar proteinosis, atypical type II pneumocytes in diffuse alveolar damage, or neoplastic cells [5-7]. In patients with relevant comorbidities and/or severely compromised lung function, the additional information obtained using BAL may be clinically relevant considering the few side effects in comparison to more invasive approaches and considering the available therapeutic options.

Surgical lung biopsy (SLB) has been considered the "gold standard" diagnostic procedure in ILDs for several years. However, 3 important points need to be clarified:

- It is not a gold standard as it samples a small part compared to the whole lung and it is still unknown which minimal quantity of lung is needed to guarantee the maximum morphological information compared to the information provided by the analysis of the whole lungs.

- Surgical lung biopsies are associated with a significant mortality at 90 days, mainly for acute exacerbation of IPF. A recent meta-analysis showed that the median diagnostic yield was 95\% (range, $42-100 \%$ ), with IPF as the most frequent diagnosis (33.5\%) [2]. Interestingly, biopsy site, biopsy number, and the SLB method were not associated with the diagnostic accuracy. The pooled postoperative mortality rate for included studies was 3.6\% (95\% confidence interval, 2.1-5.5), with significant heterogeneity observed. Given these potential risks and complications, performing a SLB is not always possible due to age, the severity of pulmonary disease, comorbidities, or patient disinclination to undergo the procedure. Therefore, in clinical practice, only about $10-15 \%$ of patients with fibrotic ILD without typical HRCT scan pattern undergo a SLB.

- Morphology, in a not negligible minority of cases, is not enough to discriminate disorders with similar histological background [3].

Transbronchial lung biopsy (TBLB) with conventional forceps is mainly useful in subjects with HRCT scan documenting alveolar opacification, bronchocentric nodules, or perilymphatic lines/nodules. The conventional forceps reach lung tissue through the bronchial routes and therefore specimens so obtained come from the centrilobular regions [4]. The disorders that are centered around terminal and respiratory bronchioles or significantly involve these structures (organizing pneumonia) or are distributed along the lymphatic routes (sarcoidosis, carcinomatous lymphangitis) may 
be easily sampled by the forceps. The role of TBLB in idiopathic interstitial pneumonias is more debatable. Berbescu et al. [8] reported that the hallmarks of usual interstitial pneumonia (UIP) (honeycombing, patchy fibrosis, and fibroblastic foci) may be identified also in TBLB samples. Tomassetti et al. [9] confirmed this observation but showed that the sensitivity of the procedure is very low and that other patterns such as nonspecific interstitial pneumonia (NSIP) and desquamative interstitial pneumonia (DIP) have a very low negative predictive value. Hence, even when these patterns are identified in tiny TBLB specimens the probability to have a diagnosis of UIP in larger samples remains high where the radiological features also suggest UIP. Transbronchial forceps biopsy may cause clinically relevant side effects, such as severe bleeding, which however occurs less often compared to other tissue sampling techniques [10].

Transbronchial lung cryobiopsy (TBLC) is a new bioptic approach in diffuse parenchymal lung diseases, and it is considered to overcome the limitations of conventional forceps biopsies. Studies on the clinical role of TBLC in ILDs are increasing and the results are promising. Samples obtained through TBLC are up to $64 \mathrm{~mm}^{2}$ in size and contain peripheral structures of the secondary pulmonary lobules [11]. The UIP pattern may be recognized on these large specimens by pathologists with high confidence and a very good interobserver variability index (Fig. 1) [12, 13]. Less high-quality evidence studies are available for other patterns such as NSIP and DIP. However, the combination of morphological information provided by TBLC and BAL profiles (a lymphocytosis in subjects with NSIP or an increase of "smoker's" macrophages and eosinophils in patients with DIP pattern) along with clinical profile and laboratory tests might be considered diagnostic and avoid a SLB. Side effects are mainly pneumothorax and bleeding. The main task of transbronchial cryobiopsy is to get good biopsy samples in size and quality whilst reducing the risk of pneumothoraces and bleeding. However, new prospective studies are still necessary to prove that TBCB is a valid substitute for SLB in a significant majority of patients with DPLD [13].

\section{TBLC: The Procedure}

\section{The Cryosurgical Equipment and Setting}

The equipment needed for TBLC is quite simple and consists of a main unit connected to a tank containing a

Transbronchial Lung Cryobiopsy in

Interstitial Lung Diseases

cooling agent, a pedal, and the probe. The entire system is easily transportable (Fig. 2).

The cryosurgical equipment works by the JouleThompson effect; hence, expansion of the gas from the compressed state leads to a change in temperature. The 


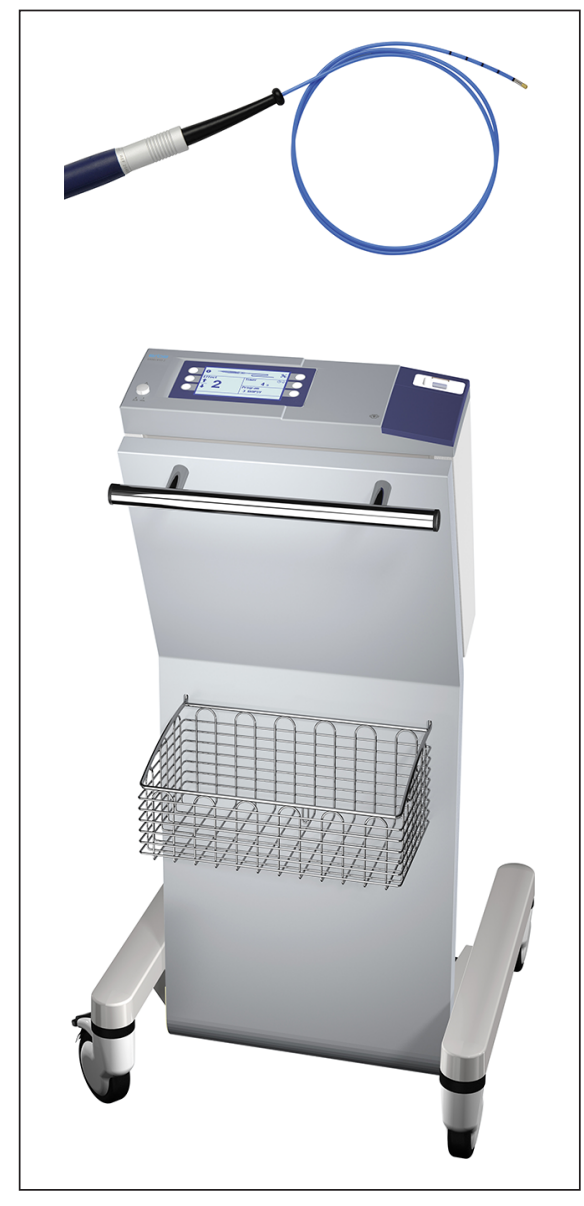

Fig. 2. The cryosurgical equipment.

cooling agent, which is mainly carbon dioxide $\left(\mathrm{CO}_{2}\right)$, is applied under high pressure through the central canal of the probe (Erbecryo II; ERBE, Tübingen, Germany). When the gas passes the end of the central canal, it expands due to the sudden difference in pressure which results in an immediate drop of temperature to less than $0{ }^{\circ} \mathrm{C}$ at the tip of the probe.

Currently, two sizes of cryoprobes are available: 1.9 and $2.4 \mathrm{~mm}$ in diameter. Both cryoprobes have a length of $900 \mathrm{~mm}$. The functionality of the probes should be tested in a water bath before taking the biopsies. This is an important check as there is no visual feedback of the freezing process when taking the biopsies in the lung periphery. Additionally, this test allows evaluation of the integrity and function of the probe tip. The freezing power of the $1.9-\mathrm{mm}$ probe is lower than that of the $2.4-\mathrm{mm}$ probe. This means, that with the $1.9-\mathrm{mm}$ probe a longer freezing time is needed in order to obtain the same sample size as with the $2.4-\mathrm{mm}$ probe. Adequate freezing time may vary not only from cryoprobe to cryoprobe, but also from patient to patient. This can be explained by different properties of the lung tissue like density or water content in different diseases. Good samples can usually be achieved with a freezing time of $7-8 \mathrm{~s}$ with the small probe and 5-6 s with the large probe using the $\mathrm{CO}_{2}$ system. There is a positive correlation between the freezing time and the size of the specimens. After having taken the first biopsies, freezing time may be adapted based on the extracted sample. Sample sizes of $5 \mathrm{~mm}$ in diameter are considered to be adequate for histological evaluation [14].

For TBLC, most centers use intubation, either with a rigid scope or a flexible tube. The rigid tracheo/bronchoscope and the endotracheal tube are the two airway devices most commonly used for TBLC. Additionally, TBLC via supraglottic devices has been described with a good safety profile [15].

\section{Sedation and Anesthesia}

The bronchoscopic procedure should be performed in deep sedation or general anesthesia in order to improve the patient's tolerance, prevent cough, and facilitate placement of the cryoprobe and the balloon, enabling optimal management of potential complications like bleeding. Usually propofol as single agent or in combination with fentanyl or remifentanil are used for deep sedation.

Patient monitoring with oxygen saturation and blood pressure is mandatory in this setting. Although even in deep sedation sufficient spontaneous ventilation is usually preserved, mechanical ventilation either with a bag or with a ventilator have to be available immediately.

\section{Where to Take a Biopsy}

The target areas for TBLC are identified by HRCT of the chest. Although there is a debate which areas should be chosen for TBLC, it seems reasonable to target different lung areas, more affected areas, and areas with different radiological patterns. This goes along with Ravaglia et al. [16] who found that taking biopsies from a different segment of the same lobe instead of biopsies from one segment rose the diagnostic yield from 69 to 96\%. However, taking biopsies only from areas suspicious for pure fibrotic changes on HRCT is unlikely to provide specific histological changes and should therefore be avoided.

\section{Safety First}

The main risk of TBLC is bleeding; therefore, precautions should always be taken in anticipation. The first safety feature is intubation. Intubation allows the flexible 

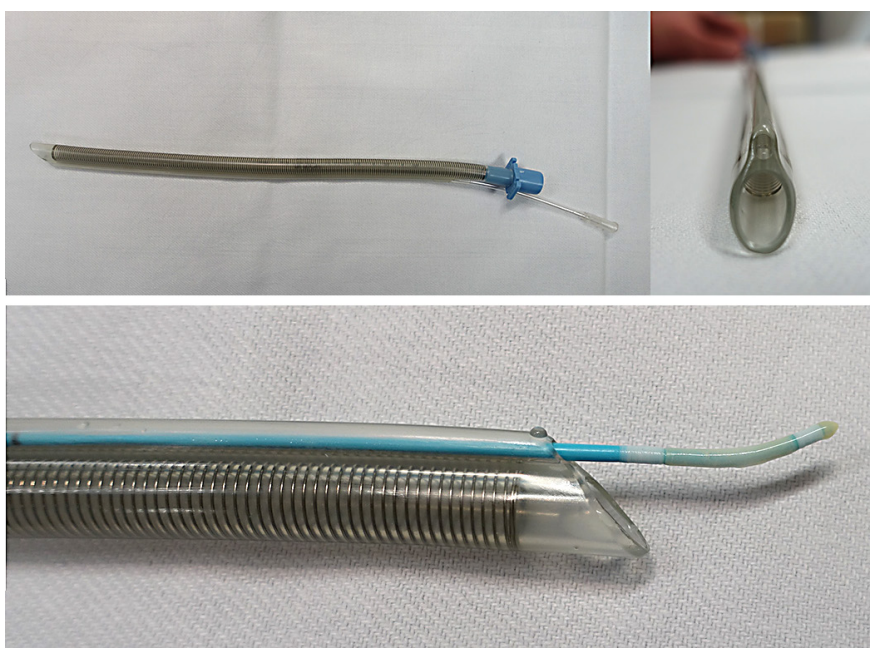

Fig. 3. Flexible tube with extra channel and angulated Fogarty balloon catheter.

bronchoscope to be removed and replaced rapidly. It allows access for large bore suction catheters and also facilitates the placement of balloon blockers. Furthermore, it is far easier to escalate ventilation strategies where the patient is already intubated.

Prophylactic endobronchial balloon placement is recommended, especially in the case of intubation with a flexible tube. The balloon should always be inflated when the flexible bronchoscope along with the cryoprobe are pulled out. The balloon is preferably placed in the segment feeding the target area. The balloon can be pushed down beside the bronchoscope within the rigid bronchoscope or through an extra channel, which is attached to the flexible tube (e.g., Bronchoflex tube Rusch; Fig. 3). Positioning of the balloon in the appropriate segment or lobe may sometimes be difficult, especially when placement in the upper lobe is needed. Some of the balloons (e.g., Fogarty balloon) offer a metallic stylet. Angulation of this stylet results in a kinking of the balloon. By rotating the balloon together with the angulated stylet, a certain degree of maneuverability can be achieved and placement is facilitated. Once in the optimal position, the balloon should be inflated with sterile water or saline (not air) with the amount of water, which is needed to occlude the bronchus completely. This determines the volume of liquid required for the prophylactic balloon inflation. From that time point on it is crucial to keep the tube/rigid scope and the balloon exactly in this defined position. If the balloon dislocates proximally into the more central airways, the injected volume of liquid is in-

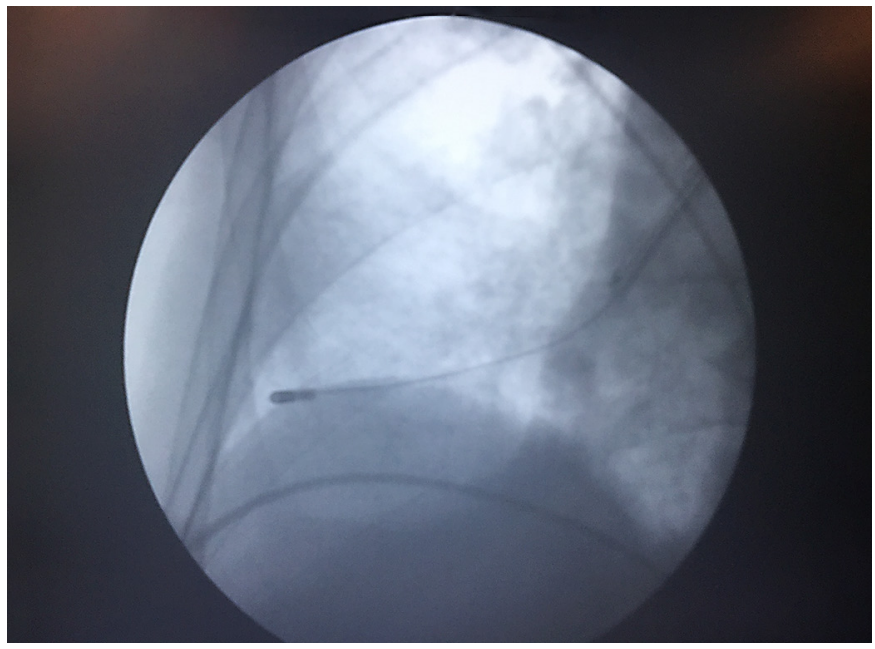

Fig. 4. The probe under fluoroscopic guidance.

sufficient for complete bronchial occlusion, thus the central airways are not protected in the case of severe bleeding.

\section{Procedural Aspects of Cryobiopsy}

The flexible bronchoscope is placed in the target lobar or segmental bronchus. Then, the cryoprobe is inserted via the working channel of the bronchoscope. Kinking of the cryoprobe should be avoided, as this may obstruct the flow of gas in the central canal within the cryoprobe, resulting in a decrease or a complete loss of the freezing power. Especially the smaller cryoprobes have to be handled very carefully, because they are less stable and kinking is more likely. The biopsies should be taken in the subpleural area. The cryoprobe is pushed down to the pleura first. The position of the cryoprobe can be rated by two criteria. First, the distance - how far is the cryoprobe pushed into the periphery? Although this varies from patient to patient depending on the size of the lung and even in an individual patient from lobe to lobe (longer distance in the lower lobe compared, e.g., to the lateral subsegment of B2), this provides useful information for the experienced endoscopist. Second and more important, fluoroscopy helps to position the probe close to the pleura. It is easier to rate the distance to the pleura when segments in which the cryoprobe is expected to be perpendicular to the chest wall (for example the lateral segment of the lower lobe) are selected. There is always a risk (mainly in subjects with bronchomalacia) that the probe cannot be pushed into the periphery of the lung, 
Table 1. Key points of TBLC

\begin{tabular}{ll}
\hline Sedation/anesthesia & General anesthesia or deep sedation (propofol/opioids) \\
Ventilation & Spontaneous breathing/mechanical ventilation/jet ventilation \\
Bronchial blocker & Different balloons, for example Fogarty balloon \\
Guidance & Fluoroscopy and length of the already pushed cryoprobe \\
Probe diameter & 1.9 or $2.4 \mathrm{~mm}$ \\
Where to take the biopsy & $\leq 1 \mathrm{~cm}$ from the pleura \\
Freezing time & Influenced by tissue properties and freezing power of the cryoprobe - aim sample \\
& of $5 \mathrm{~mm}$ in diameter \\
& $2.4 \mathrm{~mm} \times 5-6 \mathrm{~s}$ \\
& $1.9 \mathrm{~mm} \times 6-8 \mathrm{~s}$ \\
\hline
\end{tabular}

because it is stuck at a carina. Taking a biopsy in such a more central area decreases the likelihood to harvest sufficient alveolar tissue and increases the risk of severe bleeding caused by larger vessels in this area. Usually it easier to reach the subpleural area with the 1.9-mm cryoprobe, as it is more flexible and smaller. Once having reached the pleura, the probe is pulled back approximately $1 \mathrm{~cm}$, which is equivalent to the length of the metallic tip of the cryoprobe under fluoroscopic control (Fig. 4). The duration of the freezing process is determined by pressing the pedal. When the intended freezing time is reached, the probe has to be removed together with the bronchoscope in a quick movement during which, and this is crucial, the endotracheal tube and Fogarty balloon have to be fixed in their previously defined, unchanged positioning. Freezing is maintained until the probe is completely removed out of the patient. Immediately after removing the bronchoscope together with the inserted cryoprobe and its adhering tissue specimen, the preplaced balloon is inflated with the previously determined amount of water for complete bronchial occlusion. It is important not to handle the cryoprobe like forceps, i.e., pulling on the probe whilst keeping the bronchoscope in the same position will result in splashing the frozen tissue at the bronchoscope's tip. In contrast to TBLB, in TBLC the bronchoscope has to be removed out of the bronchial system together with the biopsy tool for the thawing process. This may last up to $15 \mathrm{~s}$. During that time, no bronchoscopic endobronchial control is achievable. Prophylactic balloon inflation allows to bridge this blind period by protecting the central airways in case of bleeding.

After having removed the bronchoscope together with the probe, the lung tissue, which is still attached to the probe's tip, is thawed in saline. The thawing process can be accelerated by using warm saline. The specimen should be carefully handled, minimizing tissue manipulation, because any damage of the tissue decreases its quality. Thereafter, the bronchoscope is reinserted immediately to check for bleeding. The balloon is deflated under direct visual control. If there is relevant bleeding, the balloon is inflated again for 3-10 min. It is important to carefully check every 2-3 min whether the balloon is still completely occluding the airway segment. Bleeding usually stops after this period. If bleeding persists, the balloon is reinflated for an extended period (e.g., 15-20 min). The use of intravenous tranexamic acid $1 \mathrm{~g}$ intravenously may be considered. Placement of a permanent tamponade is necessary only in rare cases.

Based on most studies, this procedure is repeated 2-4 times. However, there is no definitive answer on the recommended number of biopsies. For TBLC in different areas of the same lobe, replacement of the balloon and renewed determination of the amount of water for complete bronchial blockage are necessary. When biopsies are carried out in different lobes, 2 Fogarty balloons may be used in patients intubated with rigid tubes.

If the patient does not show symptoms suspected for pneumothorax, an expiratory chest X-ray or a thoracic sonography should be obtained $2-3 \mathrm{~h}$ after the procedure in order to exclude a pneumothorax [17].

Afterwards, fixation of the tissue in formalin is needed and samples have to be embedded with paraffin, orientating them in paraffin block in order to maximize the area for the analysis. The suggested key points of TBLC are summarized in Table 1 [18].

\section{Contraindications for TBLC}

There is limited evidence on the contraindications for the procedure. Currently, the absolute contraindications for TBLC are the presence of a bleeding diathesis, anticoagulant therapy, treatment with thienopyridines and oth- 
er new antiplatelet drugs, and thrombocytopenia with a platelet count of less than $50 \times 10^{9} / \mathrm{L}$ [7].

Pulmonary hypertension with a systolic pulmonary artery pressure $>50 \mathrm{~mm} \mathrm{Hg}$ is also considered as relative contraindication, since it may increase the risk of bleeding. The reason of an increased risk of bleeding in this context could be due to a hypertrophy of the bronchial artery. Transthoracic echocardiography is not routinely done prior to the TBLC [19]. Therefore, additional clues may be available from the CT scan of the thorax (when the pulmonary artery diameter is greater than 10 adjacent ascending aorta dimensions or when enlarged bronchial arteries may be detected). Because bleeding may also be caused by tearing pulmonary veins, areas with enlarged pulmonary veins as detected on CT scan should be avoided.

Severely compromised lung function is commonly described as relative contraindications: a FVC $<50 \%$ of predicted, a DLCO $<35 \%$, and a $\mathrm{FEV}_{1}<0.80 \mathrm{~L}$ or $<50 \%$ of predicted are exclusion criteria in some studies although not in all [19].

Several reports $[19,20]$ indicated that TBLC is safe also in patients with lower pulmonary function and in ILDs with acute or subacute behavior. However, progressive clinical and functional deterioration and the onset of ground glass areas on HRCT could be predictors of high exacerbation risk. No age limits are suggested in TBLC and this makes TBLC more feasible than SLB [19]. A list of contraindications is summarized in Table 2.

\section{Learning Curve: How to Learn TBLC}

The evidence on how learn to perform a TBLC is sparse. Ravaglia et al. [21] proposed a positive correlation of the incidence of pneumothorax with the bronchoscopist's learning curve. To our knowledge, the only study that investigated how many procedures are necessary to gain a proficiency in TBLC is by Almeida et al. [22]. They suggest that a mastery in TBLC is gained after 70 procedures, in terms of sample size, better diagnostic yield, and fewer complications.

\section{Different Approaches}

Dhooria et al. [23] reported the outcomes of TBLC carried out in different ways - with and without the fluoroscopic guidance and with and without the bronchial blocker. The study showed that fewer pneumothoraces
Table 2. Contraindications for TBLC

\section{Absolute contraindications}

Known bleeding diathesis

Anticoagulant therapy in therapeutic dosage (e.g., warfarin,

phenprocoumon, heparin, etc.)

Thienopyridines or other new antiplatelet drugs

Thrombocytopenia $\left(<50 \times 10^{9} / \mathrm{L}\right)$

\author{
Relative contraindications \\ Lung function \\ FVC $<50 \%$ of predicted \\ $\mathrm{FEV}_{1}<0.80 \mathrm{~L},<50 \%$ of predicted \\ DLCO $<35 \%$ of predicted \\ Pulmonary hypertension \\ Systolic pulmonary artery pressure $>50 \mathrm{~mm} \mathrm{Hg}$
}

and moderate-to-severe bleeding were observed using fluoroscopy and bronchial blocker, respectively [23]. Alternatively, two scopes alternated during the procedure may avoid the need for intubation [24]. In a study by Bango-Álvarez et al. [24], bleeding was controlled with this technique. Out of 106 patients, they obtained a diagnosis in 91 cases (86\%), pneumothoraces were observed in $4.7 \%$ and no acute exacerbation of IPF or severe bleeding were observed. However, it has to be kept in mind that in a case of severe bleeding it may be difficult or even impossible to enter the bronchial system with the second bronchoscope, which then results in a life-threatening situation. Previous intubation with a rigid scope or a flexible tube would provide more safety. Also, Sriprasart et al. [25] reported their experience with two scopes with no bronchial blocker but with the use of the fluoroscopy: they found a diagnostic yield of $87.84 \%$ and they reported pneumothoraces in 7\%,1 severe bleeding, and death in $4 \%$. Their mortality rate was comparable to SLB and significantly higher than that usually reported with TBLC. Hence, the authors recommend the use of either a rigid bronchoscope or endotracheal tube in conjunction with the use of a prophylactic balloon blocker with fluoroscopic guidance in order to perform the procedure in the safest manner.

\section{Outlook and Conclusions}

The future scenario that could take shape even starting from now is a significant decrease of SLB because of the relevant side effects expected, mainly in elderly or patients with comorbidities. 
Diagnostic contributors such as classical morphology, morphological information provided by the use of more sophisticated investigative tools such as biomarkers, genetic/ epigenetic signatures, and more precise interpretation of HRCT [26-31] represent a step forward in the diagnosis of ILDs and in this contest TBLC will have is significant role, provided that the quality criteria for the procedure are respected. It is crucial to reduce the probability of nondiagnostic biopsies by obtaining large samples of good quality in predefined areas and to reduce the risk of bleeding and pneumothorax by proper probe placement and taking precautions such as intubation and prophylactic balloon placement.

\section{Disclosure Statement}

Sara Colella and Maik Haentschel have no conflicts of interest. Pallav Shah reports ERBE has sponsored Imperial College for an interventional bronchoscopy course. Venerino Poletti received personal fees for talks by Boehringer Ing, Roche, and ERBE. Jürgen Hetzel received honorarium for presentation and workshop from Erbe Medizintechnik GmbH, Tübingen, Germany.

\section{References}

$>1$ Leslie KO, Gruden JF, Parish JM, Scholand MB: Transbronchial biopsy interpretation in the patient with diffuse parenchymal lung disease. Arch Pathol Lab Med 2007;131:407423.

-2 Nguyen W, Myer KC: Surgical lung biopsy for the diagnosis of interstitial lung disease: a review of the literature and recommendations for optimizing safety and efficacy. Sarcoidosis Vasc Diffuse Lung Dis 2013;30:3-16.

$\checkmark 3$ Pompeo E, Rogliani P, Cristino B, Schillaci O, Novelli G, Saltini C: Awake thoracoscopic biopsy of interstitial lung disease. Ann Thorac Surg 2013;95:445-452.

$\checkmark 4$ Poletti V, Chilosi M. Olivieri D: Diagnostic invasive procedures in diffuse infiltrative lung diseases. Respiration 2004;71:107-119.

$\checkmark 5$ Linsesen KC, Jacobs JA, Poletti V, van Mook W, Cornelissen EI, Drent M: Reactive type II pneumocytes in bronchoalveolar lavage fluid. Acta Cytol 2004;48:497-504.

6 Poletti V, Romagna M, Gasponi A, Baruzzi G, Allen KA: Bronchoalveolar lavage in the diagnosis of low-grade MALT type B cell lymphoma in the lung. Monaldi Arch Chest Dis 1995 ; 50:191-194.

$>7$ Poletti V, Romagna M, Allen KA, Spiga L: Bronchoalveolar lavage in the diagnosis of disseminated lung tumors. Acta Cytol 1995; 39:472-477.

$>8$ Berbescu EA, Katzenstein AL, Snow JL, Zisman DA: Transbronchial biopsy in usual interstitial pneumonia. Chest 2006;129:11261131.

$>9$ Tomassetti S, Cavazza A, Colby TV, Ryu JH, Nanni O, Scarpi E, Tantalocco P, Buccioli M, Dubini A, Piciucchi S, Ravaglia C, Gurioli C, Casoni GL, Gurioli C, Romagnoli M, Poletti $\mathrm{V}$ : Transbronchial biopsy is useful in predicting UIP pattern. Respir Res 2012;13:96.

10 Poletti V, Hetzel J: Transbronchial cryobiopsy in diffuse parenchymal lung diseases: need of procedural standardization. Respiration 2015;90:275-278.
11 Kropski JA, Pritchett JM, Mason WR, et al: Bronchoscopes cryobiopsy for the diagnosis of diffuse parenchymal lung disease. PLoS One 2013;8:e78674.

12 Casoni GL, Tomassetti S, Cavazza A, Colby TV, Dubini A, Ryu JH, Carretta E, Tantalocco P, Piciucchi S, Ravaglia C, Gurioli C, Romagnoli M, Gurioli C, Chilosi M, Poletti V: Transbronchial lung cryobiopsy in the diagnosis of fibrotic interstitial lung diseases. PLoS One 2014;9:e86716.

13 Tomassetti S, Wells AU, Costabel U, Cavazza A, Colby TV, Rossi G, Sverzellati N, Carloni A, Carretta E, Buccioli M, Tantalocco P, Ravaglia C, Gurioli C, Dubini A, Piciucchi S, Ryu JH, Poletti V: Bronchoscopic lung cryobiopsy increases diagnostic confidence in the multidisciplinary diagnosis of idiopathic pulmonary fibrosis. Am J Respir Crit Care Med 2016;193:745-752.

14 Colby TV, Tomassetti S, Cavazza A, Dubini A, Poletti V: Transbronchial cryobiopsy in diffuse lung disease: update for the pathologist. Arch Pathol Lab Med 2017;141:891-900.

15 Schmutz A, Dürk T, Idzko M, Koehler T, Kalbhenn J, Loop T: Feasibility of a supraglottic airway device for transbronchial lung cryobiopsy - a retrospective analysis. J Cardiothorac Vasc Anesth 2017;31:1343-1347.

16 Ravaglia C, Wells AU, Tomassetti S, Dubini A, Cavazza A, Piciucchi S, Sverzellati N, Gurioli C, Gurioli C, Costabel U, Tantalocco P, Ryu JH, Chilosi M, Poletti V: Transbronchial lung cryobiopsy in diffuse parenchymal lung disease: comparison between biopsy from 1 segment and biopsy from 2 segments - diagnostic yield and complications. Respiration 2017;93:285-292.

17 Viglietta L, Inchingolo R, Pavano C, et al: Ultrasonography for the diagnosis of pneumothorax after transbronchial lung cryobiopsy in diffuse parenchymal lung diseases. Respiration 2017;94:232-236
18 Poletti V, Ravaglia C, Dubini A, Piciucchi S, Rossi G, Kronborg-White S, Tomassetti S: How might transbronchial cryobiopsy improve diagnosis and treatment of diffuse parenchymal lung disease patients? Expert Rev Respir Med 2017;11:913-917.

19 Lentz RJ, Argento AC, Colby TV, Rickman OB, Maldonado F: Transbronchial cryobiopsy for diffuse parenchymal lung disease: a state-of-the-art review of procedural techniques, current evidence, and future challenges. J Thorac Dis 2017;9:2186-2203.

20 Hetzel J, Maldonado F, Ravaglia C, Wells AU, Colby TV, Tomassetti S, Ryu JH, Fruchter O, Piciucchi S, Dubini A, Cavazza A, Chilosi M, Sverzellati N, Valeyre D, Leduc D, Walsh SLF, Gasparini S, Hetzel M, Hagmeyer L, Haentschel M, Eberhardt R, Darwiche K, Yarmus LB, Torrego A, Krishna G, Shah PL, Annema JT, Herth FJF, Poletti V: Transbronchial cryobiopsies for the diagnosis of diffuse parenchymal lung diseases: expert statement from the cryobiopsy working group on safety and utility and a call for standardization of the procedure. Respiration 2018;95:188-200.

21 Ravaglia C, Bonifazi M, Wells AU, Tomassetti S, Gurioli C, Piciucchi S, Dubini A, Tantalocco P, Sanna S, Negri E, Tramacere I, Ventura VA, Cavazza A, Rossi A, Chilosi M, La Vecchia C, Gasparini S, Poletti V: Safety and diagnostic yield of transbronchial lung cryobiopsy in diffuse parenchymal lung diseases: a comparative study versus video-assisted thoracoscopic lung biopsy and a systematic review of the literature. Respiration 2016;91: 215-227.

22 Almeida LM, Lima B, Mota PC, Melo N, Magalhães A, Pereira JM, Moura CS, Guimarães $\mathrm{S}$, Morais A: Learning curve for transbronchial lung cryobiopsy in diffuse lung disease. Rev Port Pneumol (2006) 2017, Epub ahead of print. 
23 Dhooria S, Mehta RM, Srinivasan A, Madan K, Sehgal IS, Pattabhiraman V, Yadav P, Sivaramakrishnan M, Mohan A, Bal A, Garg M, Agarwal R: The safety and efficacy of different methods for obtaining transbronchial lung cryobiopsy in diffuse lung diseases. Clin Respir J 2017;12:1711-1720.

24 Bango-Álvarez A, Ariza-Prota M, Torres-Rivas $\mathrm{H}$, Fernández-Fernández L, Prieto A, Sánchez I, Gil M, Pando-Sandoval A: Transbronchial cryobiopsy in interstitial lung disease: experience in 106 cases - how to do it. ERJ Open Res 2017;300148-2016.

25 Sriprasart T, Aragaki A, Baughman R, Wikenheiser-Brokamp K, Khanna G, Tanase D, Kirschner M, Benzaquen S: A single US center experience of transbronchial lung cryobiopsy for diagnosing interstitial lung disease with a 2 -scope technique. J Bronchology Interv Pulmonol 2017;24:131-135.
26 Hernández-González F, Lucena CM, Ramírez J, Sánchez M, Jimenez MJ, Xaubet A, Sellares J, Agustí C: Cryobiopsy in the diagnosis of diffuse interstitial lung disease: yield and costeffectiveness analysis. Arch Bronconeumol 2015;51:261-267.

27 Kim SY, Diggans J, Pankratz D, et al: Classification of usual interstitial pneumonia in patients with interstitial lung disease: assessment of a machine learning approach using high-dimensional transcriptional data. Lancet Respir Med 2015;3:473-482.

28 Kim SY, Diggans J, Pankratz D, Huang J, Pagan M, Sindy N, Tom E, Anderson J, Choi Y, Lynch DA, Steele MP, Flaherty KR, Brown KK, Farah H, Bukstein MJ, Pardo A, Selman M, Wolters PJ, Nathan SD, Colby TV, Myers JL, Katzenstein AL, Raghu G, Kennedy GC: Longitudinal change in collagen degradation biomarkers in idiopathic pulmonary fibrosis: an analysis from the prospective, multicentre PROFILE study. Lancet Respir Med 2015;3:462-472.
29 Wells AU, Costabel U, Poletti V, Crestani B, Egan J, Margaritopoulos G, Antoniou K: Challenges in IPF diagnosis, current management and future perspectives. Sarcoidosis Vasc Diffuse Lung Dis 2015;32 Suppl 1:28-35.

30 Kropski JA, Blackwell TS, Loyd JE: The genetic basis of idiopathic pulmonary fibrosis. Eur Respir J 2015;45:1717-1727.

31 Gruden JF, Panse PM, Leslie KO, Tazelaar HD, Colby TV: UIP diagnosed at surgical lung biopsy, 2000-2009: HRCT patterns and proposed classification system. AJR Am J Roentgenol 2013;200:W458-W467. 